as tissue, bone and a void (in the sinuses). This can lead to artefacts in the images that must be accounted for during image analysis, says Gordon.

Currently, 3 T MRI systems are the standard for high-end human neuroimaging. But research-grade 7 T MRI instruments for human studies have come a long way in recent years with several companies, including Siemens Medical Solutions, GE Healthcare in Piscataway, New Jersey, and Philips in Andover, Massachusetts, now supplying second-generation versions of these systems. "The first generation of $7 \mathrm{~T}$ systems were monsters that needed 400 tonnes of shielding, but the second-generation systems are actively shielded so now in principle you don't need any iron to shield the magnet," says Gordon. But many researchers say that, even with the advances, $7 \mathrm{~T}$ systems still need more engineering work.

\section{The 'best' field strength?}

"The $7 \mathrm{~T}$ scanner is still a bit of a specialized device and, in my opinion, has yet to reach its full potential," says Larry Wald, director of the NMR core facility at the Athinoula A. Martinos Center for Biomedical Imaging in Charlestown, Massachusetts. Deshpande agrees: "There will be some ramp-up time with

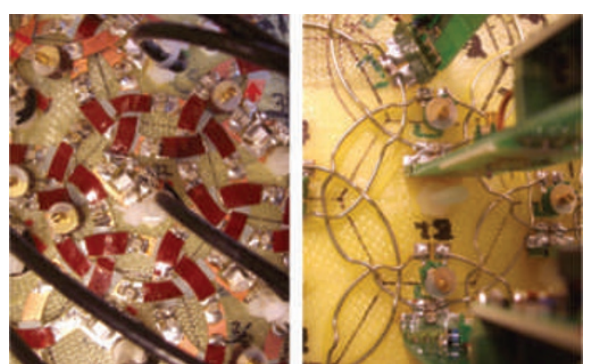

The radio-frequency coils used to transmit and detect MRI signals are being made more sensitive.

the $7 \mathrm{~T}$ in terms of applications and research. We need to find out how far we can push the instrument."

Whether $7 \mathrm{~T}$ will eventually become a clinically robust field strength for human MRI is not clear. "Ever since the beginning of MRI there has always been discussion about what field one should work at," says Koretsky. First there was the decision between using $0.5 \mathrm{~T}$ and $1.5 \mathrm{~T}$, and then between $3 \mathrm{~T}$ and $4 \mathrm{~T}$ magnets. Now, he says, a $7 \mathrm{~T}$ debate might start. "Some of the images from the brain at $7 \mathrm{~T}$ are truly amazing," says Deshpande. "We are seeing things that we have never seen at either $1.5 \mathrm{~T}$ or $3 \mathrm{~T}$."

Even as new $7 \mathrm{~T}$ human systems and $11.7 \mathrm{~T}$ and higher animal systems from companies such as Bruker Biospin and Varian in Palo Alto, California, expand in use within the neuroscience community, bigger magnets are being designed for cutting-edge animal and human imaging. In autumn 2008, the Martinos Center installed a $15 \mathrm{~T}$ magnet designed by Varian and Magnex Scientific in Walnut Creek, California, for imaging mice and rats, which Wald says is now at field strength and should be generating its first images in the coming weeks. The machine, used in conjunction with knockout mice with genes that have been 'turned off', should allow scientists to understand neural disease progression more effectively and even test potential drug therapies, says Wald.

Field strengths for human MRI magnets may reach double digits in the coming years. Both the NIH and NeuroSpin, a centre for ultra-highfield MRI in Saclay, France, have announced projects to construct 11.7 T MRI magnets for imaging human subjects. Although Koretsky says that it will be several years before these new magnets are installed and operational, he thinks the images will be worth the wait. "In the end you would love an anatomical picture that looks like a histological slice," he says, adding that these new magnets will get them closer than ever to making this a reality.

\title{
CHANGING THE COLOUR OF MRI
}

When Alan Koretsky, scientific director of the National Institute of Neurological Disorders and Stroke in Bethesda, Maryland, and Gary Zabow began to think about developing new contrast agents for magnetic resonance imaging (MRI), they took their design cues from the colourful world of molecular imaging. "It was looking at what existed and then figuring out some way to copy the idea of quantum dots," says Zabow, a physicist at the National Institute of Standards and Technology in Boulder, Colorado.

In molecular imaging, a quantum dot can generate a range of possible emission spectra simply by varying the size of the dot's inner core shell. This is a stark contrast to the traditional agents used in MRI, such as gadolinium or iron oxide, which are magnetic materials that alter the signal from the protons in the surrounding water, appearing as either darker or brighter spots on images. "It is sometimes difficult to tell the different agents apart from one another or from artefacts that make something brighter or darker," says Zabow. (At present, colour in MRI scans - such as those in this article - is assigned to shades of grey and added during processing.)

"One of the areas of MRI that has exploded over the past 5 or 6 years is the ability to track cells as they move around," says Koretsky. Although MRI cannot achieve single-cell resolution, a single cell can have sufficient magnetic-resonance contrast to be detected. But for researchers interested in tracking two or three cells at once, this level of differentiation is not enough.

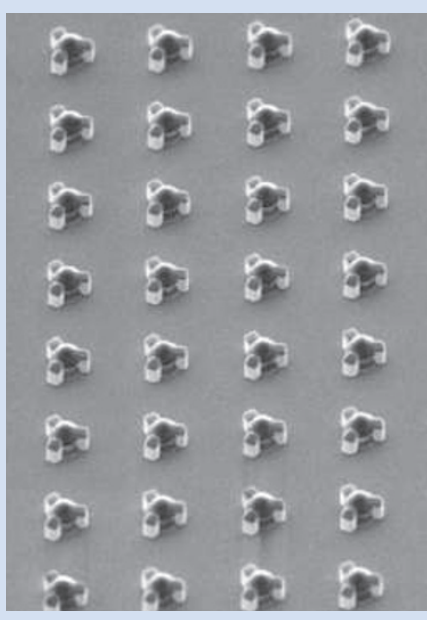

Electron-microscopy images of microfabricated contrast agents.
So Zabow and Koretsky microfabricated specific magnetic shapes that would create different magnetic fields and so shift the nuclear magnetic resonance frequency. "The existing magnetic particles do not shift the frequency - they just broaden it out," says Zabow. But the very precise shape of these new agents generates a corresponding precise frequency shift, similar to quantum dots, giving Zabow and Koretsky the possibility of creating different colours through different shapes and their specific frequency shifts.

The initial work consists of two discs with a gap between them in which the magnetic field can be generated. By varying the thickness or diameter of the discs, or the gap, different fields can be obtained so that when water passes between the discs the magnetic resonance of the water molecules flowing through the gap shifts (G. Zabow et al. Nature 453, 1058-1063; 2008).

Although the possible range of new colours is still to be determined, that is not the primary focus at the moment, says Zabow. They are working to improve the

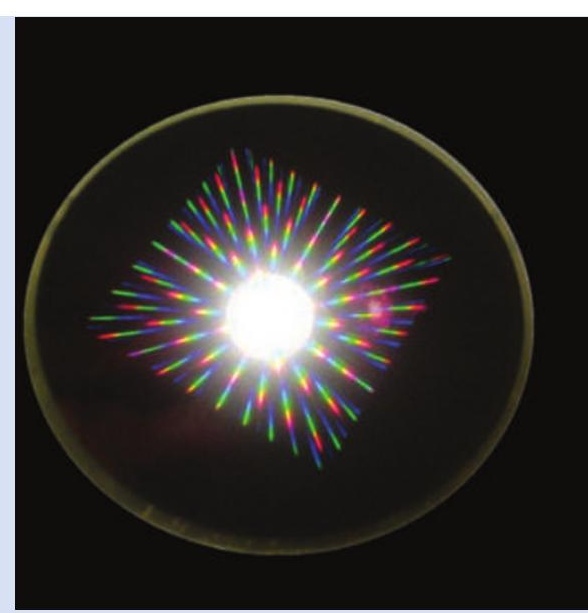

Microfabricated shapes provide potential for a wide range of possible colours when used in MRI.

fabrication process and make the magnetic particles smaller and more robust. "We are working first on that. The idea of having as many colours as possible falls out from there because in improving the fabrication we are getting the geometry more precise," he says.

Koretsky sees these new contrasting agents as adding a unique ability to MRI that no other radiological imaging technique possesses. 